\title{
LIFETIME MAXIMIZATION FOR BEAMFORMING APPLICATIONS IN WIRELESS SENSOR NETWORKS
}

\author{
Benjamín Béjar, Santiago Zazo* \\ Universidad Politécnica de Madrid \\ ETSI de Telecomunicación, C-303, 28080, Madrid, Spain \\ e-mail: \{min, santiago\}@gaps.ssr.upm.es
}

\author{
Daniel P. Palomar \\ The Hong Kong University of Science and Technology \\ ECE Department, Clear Water Bay, Hong Kong SAR \\ e-mail: palomar@ust.hk
}

\begin{abstract}
Energy efficiency is a major design issue in the context of Wireless Sensor Networks (WSN). If data is to be sent to a far-away base station, collaborative beamforming by the sensors may help to distribute the load among the nodes and reduce fast battery depletion. However, collaborative beamforming techniques are far from optimality and in many cases may be wasting more power than required.

In this contribution we consider the issue of energy efficiency in beamforming applications. Using a convex optimization framework, we propose the design of a virtual beamformer that maximizes the network's lifetime while satisfying a pre-specified Quality of Service (QoS) requirement. A distributed consensus-based algorithm for the computation of the optimal beamformer is also provided.
\end{abstract}

Index Terms - Energy-efficiency, consensus, beamforming

\section{INTRODUCTION}

Beamforming techniques adjust the antenna weights in order to mitigate fading channel or interference effects, thus enhancing the quality of the signal of interest. In the context of a Wireless Sensor Network (WSN), it may happen that the area of interest to be sensed is located in a remote region of difficult access. To overcome the problem of retrieving the gathered data, nodes can cooperate to form a virtual beamformer in order to send the acquired data to a far-away base station for further processing and analysis. A certain Quality of Service (QoS) measure must be imposed at the receiver side (i.e. base station) that allows reliable signal decoding.

One possible solution to this end is the concept of collaborative beamforming [1] where nodes synchronize their phases to add constructively at the base station. The statistical properties of the average radiation pattern have been analyzed for different distributions of the nodes [1-4]. It is demonstrated that as the number of nodes increases the average directivity of the virtual array approaches its maximum. Although the average properties of the radiation pattern are insightful they only hold asymptotically when the number of nodes is very large. Further, channel effects are usually ignored and in many situations we may be wasting more power than necessary (far from optimality). In order to meet some QoS at the receiver it would be more energy-efficient to optimize the individual antenna weights so as to maximize the network's lifetime, using the more

${ }^{*}$ This work was supported in part by the Spanish Ministry of Science and Innovation under the grant TEC2009-14219-C03-01; El Consejo Social de la UPM; the Spanish Ministry of Science and Innovation in the program CONSOLIDER-INGENIO 2010 under the grant CSD2008-00010 COMONSENS; Spanish Ministry of Science and Innovation under the grant TEC2010-21217-C02-02-CR4HFDVL; The Hong Kong RGC 617810 research grant. mature beamforming technology for centralized scenarios, otherwise we may cause rapid energy depletion at the nodes, shortening their time of activity.

In the last few years, the application of convex optimization techniques to beamforming problems has been proven very successful, see [5] and references therein. The use of convex optimization can help to produce optimal or close to optimal solutions in many beamforming problems. In the context of WSN, energy efficiency is a major design issue and there has been little attention to this issue in the context of beamforming applications. In $[6,7]$ the issue of energy-efficiency is considered when collaborative beamforming is used. However, both works are oriented to routing optimization instead of energy efficient beamforming. The development of distributed optimization techniques that take into account energy efficiency are of paramount importance in WSN's.

We consider the distributed beamforming problem with QoS constraints where the metric to be optimized is the network's lifetime (i.e. the time that the network can guarantee the specified QoS requirement). We derive closed-form expressions for the optimal beamformer and provide an iterative algorithm for its numerical computation. Using only local information about battery status and channel conditions, we use consensus [8] to find a fully distributed version of the centralized algorithm (i.e. only require local communication among nodes).

\section{SYSTEM MODEL}

Consider a WSN composed of $M$ battery-powered single-antenna nodes scattered over a certain area and the following discrete-time communication system

$$
y[k]=\mathbf{w}^{\mathrm{H}}[k] \mathbf{h} s[k]+n[k],
$$

where $y[k]$ is the received signal at time instant $k, \mathbf{h}=\left[h_{1}, \ldots, h_{M}\right]^{\top}$ is the channel between the nodes and the base station, $\mathbf{w}[k]=$ $\left[w_{1}, \ldots, M\right]$ is the beam-vector, $s[k]$ is the discrete-time signal to be transmitted and $n[k]$ represents the measurement noise process. The noise samples $n[k]$ are assumed to be independent and identically (i.i.d.) distributed Gaussian random variables of zero mean and variance $\sigma_{n}^{2}$, that is $n[k] \sim \mathcal{N}\left(0, \sigma_{n}^{2}\right)$.

The (instantaneous) received Signal to Noise Ratio (SNR) at destination is given by $\Gamma[k]=\left|\mathbf{w}^{\mathrm{H}}[k] \mathbf{h}\right|^{2} \frac{P_{s}}{\sigma_{n}^{2}}=\left|\mathbf{w}^{\mathrm{H}}[k] \mathbf{h}\right|^{2} \rho_{0}$, where expectation is taken over the noise $n[k]$ and the symbols $s[k]$, with $P_{s}=\mathrm{E}\left[|s[k]|^{2}\right], \mathrm{E}[s[k]]=0$ and $\rho_{0}=P_{s} / \sigma_{n}^{2}$.

We will use the instantaneous received SNR $\Gamma[k]$ as our QoS measure, i.e. $\Gamma[k] \geq \rho$. Besides, we also seek to maximize the network's lifetime so that it can be operative for the largest period of time. Several measures of network's lifetime have been proposed in 


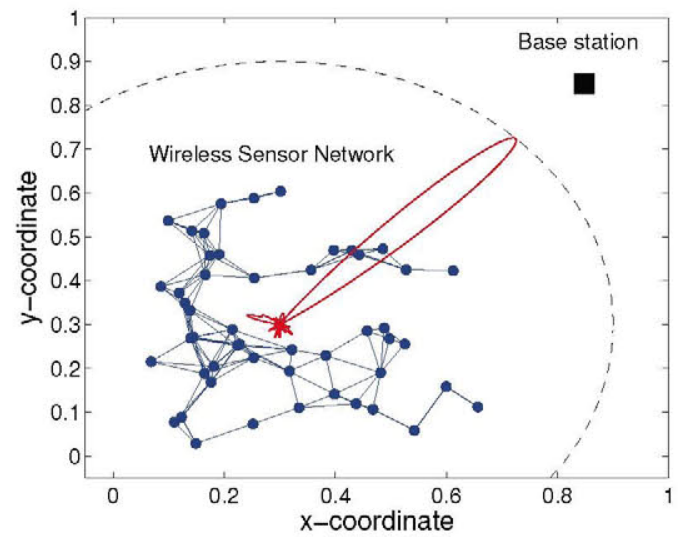

Fig. 1: Beamforming scenario between the nodes and the far-away base station.

the literature (see [9] and references therein) attending to different criteria like percentage of alive nodes, coverage area or connectivity, among others. In our problem a natural measure of the network's lifetime is the time that the network can satisfy the QoS constraint. We will show later that such lifetime criterion is equivalent, in our system model, to maximizing the time for the first node to deplete its battery.

\section{ENERGY-EFFICIENT BEAMFORMING}

Let $E_{m}$ denote the initial battery level of node $m$. The amount of energy consumed during the $k$-th sampling period at node $m$ would be $\left|w_{m}[k]\right|^{2} T_{\mathrm{s}}$, where $T_{s}$ is the sampling period.

Definition 1 (Deterministic network lifetime). The lifetime of the network is the time that the QoS constraint $(\Gamma[k] \geq \rho)$ can be satisfied.

Our goal is then to find the sequence of beamforming vectors $\{\mathbf{w}[k]\}$ that maximize that network's lifetime given in Definition 1. As nodes are battery-equipped elements with limited power resources, we also impose a maximum transmission power $p_{m}$ on each node.

Let denote $K^{\star}$ as the maximum time that the QoS constraint can be satisfied, then the problem of finding the optimal beam-vectors $\left\{\mathbf{w}[1], \ldots, \mathbf{w}\left[K^{\star}\right]\right\}$, can be expressed as

$$
\begin{array}{lll}
\text { find } & \left\{\mathbf{w}[1], \ldots, \mathbf{w}\left[K^{\star}\right]\right\} \in \mathbb{C}^{M} & \\
\text { subject to } & \left|\mathbf{w}^{\mathrm{H}}[k] \mathbf{h}\right|^{2} \rho_{0} \geq \rho & k=1, \ldots, K^{\star} \\
& \left|w_{m}[k]\right|^{2} \leq p_{m} & \text { for all } m, k \\
& T_{\mathrm{s}} \sum_{k=1}^{K^{\star}}\left|w_{m}[k]\right|^{2} \leq E_{m} & m=1, \ldots, M
\end{array}
$$

where the last constraint ensures that no node can waste more energy than its actual battery level.

Although the time index $k$ is discrete, we will consider it as a continuous variable in the optimization process. Based on problem (2) we can establish the following result:

Lemma 1. Let $\left\{\mathbf{w}^{\star}[1], \ldots, \mathbf{w}^{\star}\left[K^{\star}\right]\right\}$ be a solution (feasible point) of the feasibility problem (2). Then, the set of vectors $\mathbf{z}[k], k=$ $1, \ldots, K$ with $\left|z_{m}[k]\right|=\left|w_{m}^{\star}[k]\right|$ and $\angle z_{m}[k]=\angle h_{m}$ are also optimal.
Proof. Since $\left|z_{m}[k]\right|=\left|w_{m}^{\star}[k]\right|$ for all $m$ and $k$, the second and third constraints of problem (2) are automatically satisfied as they only involve the magnitude of $w_{m}[k]$. We only need to show that the first constraint is also satisfied. We then have that $\rho \leq \rho_{0}\left|\sum_{m=1}^{M}\left(w_{m}^{\star}[k]\right)^{*} h_{m}\right|^{2} \leq \rho_{0}\left(\sum_{m=1}^{M}\left|w_{m}^{\star}[k]\right|\left|h_{m}\right|\right)^{2}=$ $\rho_{0}\left|\mathbf{z}^{\mathrm{H}}[k] \mathbf{h}\right|^{2}$, and the result follows.

Let $\bar{w}_{m}[k]=\mid w_{m}\left[k||\right.$ and $\bar{h}_{m}=\left|h_{m}\right|$, i.e. the magnitude of the $m$-th beam-weight and the $m$-th channel coefficient, respectively. Based on Lemma 1 we could fix the phase of the beam-vectors $\left\{\mathbf{w}[1], \ldots, \mathbf{w}\left[K^{\star}\right]\right\}$ to match that of the channel and replace problem (2) by the following (real-valued) feasibility power allocation problem

$$
\begin{array}{lll}
\text { find } & \left\{\overline{\mathbf{w}}[1], \ldots, \overline{\mathbf{w}}\left[K^{\star}\right]\right\} \in \mathbb{R}_{+}^{M} & \\
\text { subject to } & \overline{\mathbf{w}}^{\top}[k] \overline{\mathbf{h}} \geq \sqrt{\rho / \rho_{0}} & k=1, \ldots, K^{\star} \\
& \bar{w}_{m}^{2}[k] \leq p_{m} & \text { for all } m, k \\
& T_{\mathrm{s}} \sum_{k=1}^{K^{\star}} \bar{w}_{m}^{2}[k] \leq E_{m} & m=1, \ldots, M
\end{array}
$$

where $\overline{\mathbf{w}}[k]=\left[\bar{w}_{1}[k], \ldots, \bar{w}_{M}[k]\right]^{\top}$ and $\overline{\mathbf{h}}=\left[\bar{h}_{1}, \ldots, \bar{h}_{M}\right]^{\top}$. Note that feasibility problem (3) is convex and hence, can be solved efficiently. Further, since the problem is convex it can be easily shown that an optimal constant beam-vector (independent of time) exists.

Lemma 2. Assume that feasibility problem (3) is feasible. Then, there exist an optimal solution $\left\{\overline{\mathbf{w}}^{\star}[1], \ldots, \overline{\mathbf{w}}^{\star}\left[K^{\star}\right]\right\}$ to (3) such that $\overline{\mathbf{w}}^{\star}[i]=\mathbf{w}^{\star}$ for all $i=1, \ldots, K^{\star}$.

Proof. Assume that $\left\{\overline{\mathbf{w}}^{\star}[1], \ldots, \overline{\mathbf{w}}^{\star}\left[K^{\star}\right]\right\}$ is a solution of the feasibility problem (3). Then the constant sequence with elements equal to $\overline{\mathbf{w}}^{\star}=\min _{i}\left\|\overline{\mathbf{w}}^{\star}[i]\right\|, i=1, \ldots, K^{\star}$, is also a feasible point (sequence).

However, we still need to compute the optimal time $K^{\star}$ in order to compute the optimal beamvector. Since, by Lemma 2 , for any $K^{\star}$ there exist always a constant beamvector that solves problem (3), then we can formulate the lifetime maximization problem as:

$$
\begin{array}{lll}
\underset{\mathbf{w} \geq 0, K}{\operatorname{maximize}} & K & \\
\text { subject to } & \overline{\mathbf{w}}^{\top} \overline{\mathbf{h}} \geq \sqrt{\rho / \rho_{0}} & \\
& \bar{w}_{m}^{2} \leq p_{m} & m=1, \ldots, M \\
& K \bar{w}_{m}^{2} T_{\mathrm{s}} \leq E_{m} & m=1, \ldots, M
\end{array}
$$

Note that the last constraint of problem (4) is not convex. However, by an appropriate change of variables it can be transformed into convex form. Consider the change of variable $t=1 / K$, then we can reformulate problem (4) as the following equivalent minimization problem:

$$
\begin{aligned}
& \underset{\overline{\mathbf{w}} \geq 0, t}{\operatorname{minimize}} \quad t \\
& \text { subject to } \quad \overline{\mathbf{w}}^{\top} \overline{\mathbf{h}} \geq \sqrt{\rho / \rho_{0}} \\
& \begin{array}{ll}
\bar{w}_{m}^{2} \leq p_{m} & m=1, \ldots, M \\
\bar{w}_{m}^{2} \leq t E_{m} / T_{\mathrm{s}} & m=1, \ldots, M
\end{array}
\end{aligned}
$$

where now the last constraint of (5) corresponds to a second order cone constraint and hence, it's convex and can be solved efficiently.

As we mentioned earlier, it turns out that, in our particular setting, maximizing for the QoS lifetime criterion (i.e. Definition 1) coincides with maximizing the time for the first node to deplete its battery (i.e. 1st node depletion criterion). To see the equivalence note that the forecasted longevity of a node will be given by 
$E_{m} /\left(\left|w_{m}\right|^{2} T_{s}\right)$. It can be easily shown that the maximization of the minimum node longevity can be expressed as

$$
\begin{array}{lll}
\underset{\overline{\mathbf{w}} \geq 0}{\operatorname{minimize}} & \max \left(\frac{\bar{w}_{1}^{2} T_{\mathrm{s}}}{E_{1}}, \ldots, \frac{\bar{w}_{M}^{2} T_{\mathrm{s}}}{E_{M}}\right) & \\
\text { subject to } & \overline{\mathbf{w}}^{\top} \overline{\mathbf{h}} \geq \sqrt{\rho / \rho_{0}} & \\
& \bar{w}_{m}^{2} \leq p_{m} & m=1, \ldots, M
\end{array}
$$

It is easy to see that problem (5) is the epigraph form of problem (6).

Proposition 1. Suppose problem (5) is solvable, then the optimal power allocation is given by

$$
\begin{gathered}
\bar{w}_{m}^{\star}=\min \left(\sqrt{\frac{t^{\star} E_{m}}{T_{s}}}, \sqrt{p_{m}}\right), \\
t^{\star}=\left(\frac{\sqrt{\rho / \rho_{0}}-\sum_{m \notin \mathcal{M}}\left|h_{m}\right| \sqrt{p_{m}}}{\sum_{m \in \mathcal{M}}\left|h_{m}\right| \sqrt{E_{m} / T_{s}}}\right)^{2}
\end{gathered}
$$

where $\mathcal{M}=\left\{\left.m|| \bar{w}_{m}\right|^{2}<p_{m}\right\}$ is the set of nodes not transmitting at maximum power.

Proof. The Lagrangian of (5) is

$$
\mathcal{L}=t\left(1-\boldsymbol{\mu}^{\top} \mathrm{e}\right)+\overline{\mathbf{w}}^{\top} \Delta \overline{\mathbf{w}}-\lambda \overline{\mathbf{h}}^{\top} \overline{\mathbf{w}}+\lambda \sqrt{\frac{\rho}{\rho_{0}}}-\boldsymbol{\sigma}^{\top} \mathrm{p}
$$

where $\boldsymbol{\mu}=\left[\mu_{1}, \ldots, \mu_{M}\right]^{\top}, \boldsymbol{\sigma}=\left[\sigma_{1}, \ldots, \sigma_{M}\right]^{\top}, \lambda, \mu_{m}, \sigma_{m} \in \mathbb{R}_{+}$ are the associated Lagrange multipliers, $\mathrm{e}=\frac{1}{T_{\mathrm{s}}}\left[E_{1}, \ldots, E_{M}\right]^{\top}$, $\mathbf{p}=\left[p_{1}, \ldots, p_{M}\right]^{\top}$ and $\Delta=\operatorname{diag}(\boldsymbol{\mu}+\boldsymbol{\sigma})$. The Karush-KuhnTucker (KKT) conditions are then given by

$$
\begin{array}{rlrlrl}
\bar{w}_{m}^{2}-p_{m} & \leq 0, & \sigma_{m}\left(\bar{w}_{m}^{2}-p_{m}\right) & =0 & & 2 \Delta \overline{\mathbf{w}}=\lambda \overline{\mathbf{h}} \\
\overline{\mathbf{w}}^{\top} \overline{\mathbf{h}}-\sqrt{\rho / \rho_{0}} \geq 0, & \lambda\left(\sqrt{\rho / \rho_{0}}-\overline{\mathbf{w}}^{\top} \overline{\mathbf{h}}\right) & =0 & \boldsymbol{\mu}^{\top} \mathrm{e}=1 \\
\bar{w}_{m}^{2}-t E_{m} / T_{\mathrm{s}} \leq 0, & \mu_{m}\left(\bar{w}_{m}^{2}-t E_{m} / T_{\mathrm{s}}\right) & =0 &
\end{array}
$$

Assume that $\bar{w}_{m}<\sqrt{p_{m}}$, we then have that $\sigma_{m}=0$. We also know that all nodes must be active (i.e. $\bar{w}_{m}>0$ ) which implies that $\mu_{m}>0$ which further implies $\bar{w}_{m}=\sqrt{t E_{m} / T_{s}}$ and that $\sqrt{\rho / \rho_{0}}=\overline{\mathbf{w}}^{\top} \overline{\mathbf{h}}$. Since $w_{m}^{2} \leq p_{m}$ we have

$$
\overline{\mathbf{w}}^{\top} \mathbf{h}=\sum_{m=1}^{M} \min \left(\sqrt{t^{\star} E_{m} / T_{s}}, \sqrt{p_{m}}\right)\left|h_{m}\right|=\sqrt{\rho / \rho_{0}}
$$

which is equivalent to

$$
\sum_{m \in \mathcal{M}} \sqrt{t^{\star} E_{m} / T_{\S}}\left|h_{m}\right|+\sum_{m \notin \mathcal{M}} \sqrt{p_{m}}\left|h_{m}\right|=\sqrt{\rho / \rho_{0}} .
$$

Solving the above equation for $t^{\star}$ leads to (8).

It is immediate to realize that the optimal beam-vector is $\left(\mathbf{w}^{\star}\right)^{\top}=\left[\bar{w}_{1}^{\star} h_{1} /\left|h_{1}\right|, \ldots, \bar{w}_{M}^{\star} h_{M} /\left|h_{M}\right|\right]$. From the closed-form expressions (7) and (8) we also realize that, at the optimum, all nodes must be active (i.e. $\bar{w}_{m}>0$ for all $m$ ). Further, since all nodes transmitting below its maximum allowed transmission power have the same ratio $E_{m} /\left|w_{m}^{\star}\right|^{2}, m \in \mathcal{M}$ (i.e. share the same value of $t^{\star}$ ), then they will deplete their batteries at the same time.

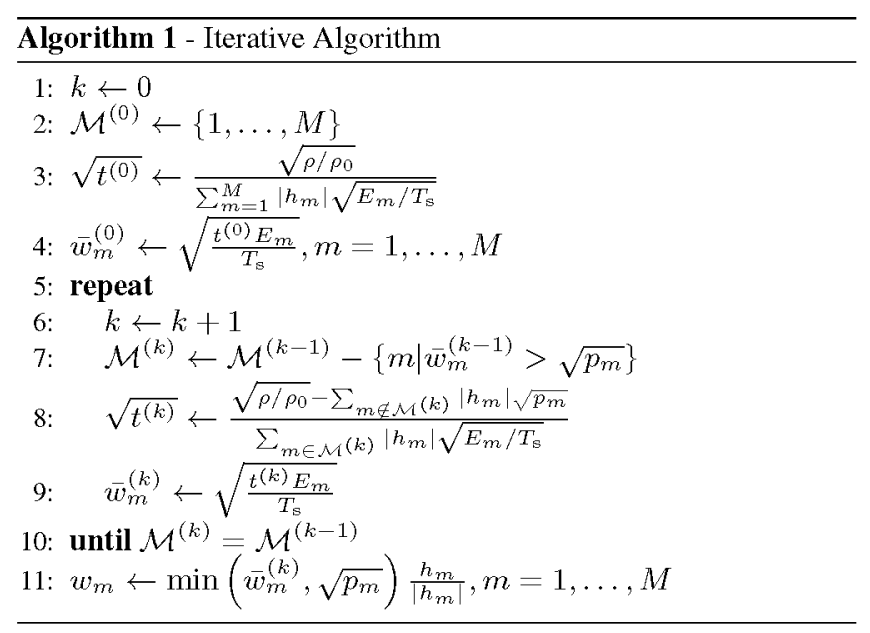

\section{ALGORITHMS}

In this section we present an iterative algorithm for the computation of the optimal beamformer of problem (5) using the closed form expressions (7) and (8). The procedure is summarized in Algorithm 1. By matching the phase of the beam-vector to that of the channel we obtain a solution to the original beamforming problem (2). It can be shown that Algorithm 1 converges to the optimal beamformer. However, due to space limitations we skip the details here. The problem with Algorithm 1 is that it is centralized and therefore, it is not practical in the context of WSNs. However, by inspection of Algorithm 1 it is easy to realize that a distributed counterpart based on consensus is possible. The idea is very simple and is based on the observation that $t^{(k)}$ in step 8 of Algorithm 1 can be obtained by dividing two terms, each of which can be computed by means of consensus. To that end, consider two variables per node $\gamma_{m}$ and $\beta_{m}$, the first one contributing to the numerator and the latter one to the denominator of (8). Initially, we assume that all nodes are trans-

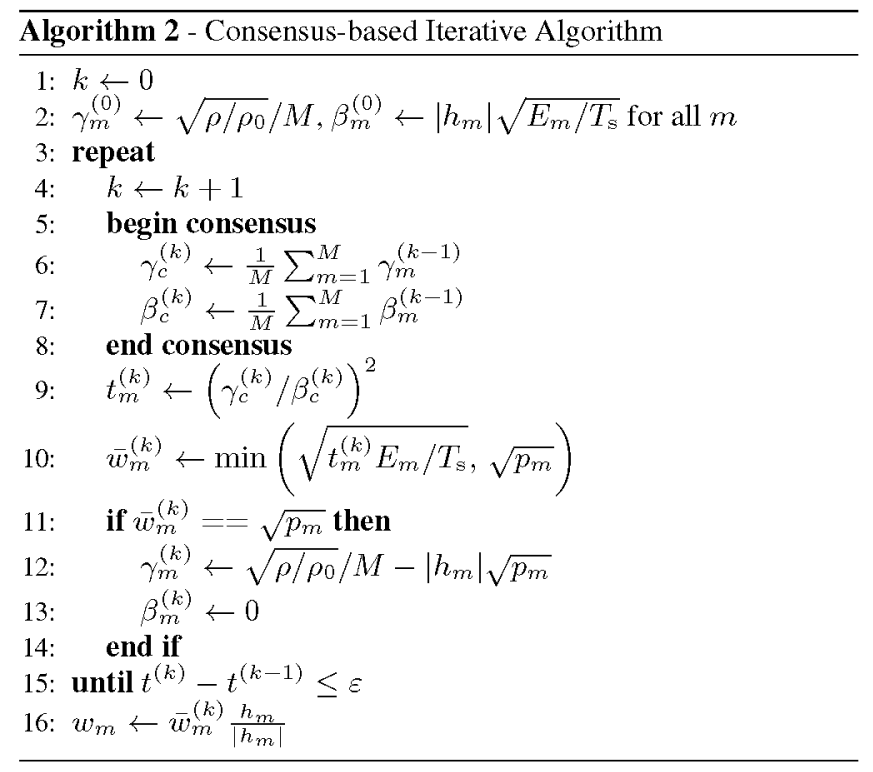

mitting below their maximum power (i.e. the same as in Algorithm 1) so that $\gamma_{m}=\sqrt{\rho / \rho_{0}} / M$ and $\beta=\left|h_{m}\right| \sqrt{E_{m} / T_{s}}$. If we perform an average consensus over these two quantities, they converge to 
$\frac{1}{M} \sum_{m} \gamma_{m}$ and $\frac{1}{M} \sum_{m} \beta_{m}$ at each node [8]. By dividing these two average quantities we get the same value of $t$ as in the centralized Algorithm 1. After that, each node computes its power allocation $v_{m}$ as in (7). If a node is required to transmit at its maximum power then, it sets $\gamma_{m}=\sqrt{\rho / \rho_{0}} / M-\left|h_{m}\right| \sqrt{p_{m}}$ and $\beta_{m}=0$ for the next iteration. The complete algorithm is shown in Algorithm 2. It is easy to see that the two Algorithms 1 and 2 yield the same solution.

\section{NUMERICAL SIMULATIONS}

In this section we provide some numerical results in order to illustrate the proposed approach and algorithms. We have generated a random network of $M=50$ nodes uniformly distributed in a unit square. Connectivity among nodes has been set based on a coverage radius criterion with an average degree of 4 . The symbol to background noise power ratio $\rho_{0}=P_{s} / \sigma_{n}^{2}$ has been set to $20 \mathrm{~dB}$. Nodes have an initial random battery level uniformly distributed within the interval $[0.51]$. The channel coefficients follow a circularly symmetric complex Gaussian distribution of zero mean and unit variance (i.e. Rayleigh fading).

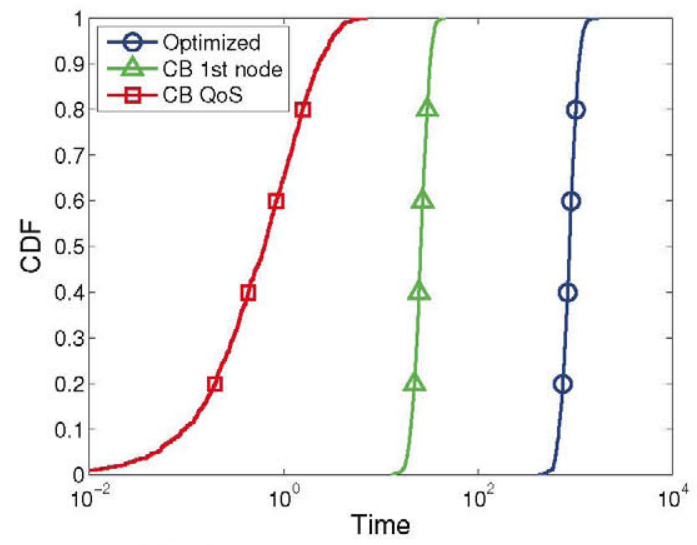

Fig. 2: Lifetime CDF for $\rho=20 \mathrm{~dB}$

We have compared the proposed energy-aware lifetime optimization method with a collaborative beamforming (CB) strategy that adjust the power of the nodes (i.e. the same for all nodes) in order to meet the QoS constraint. In Figure 2 we have plotted the CDF of the network lifetime based on 1000 realizations for a target QoS $\rho=20 \mathrm{~dB}$. For the CB strategy we have represented the time at which the first node deplete its battery (CB 1st node) and the time for which the QoS can be guaranteed (CB QoS). With the proposed approach we can improve the lifetime of the network by more than one order of magnitude.

In Figure 3-a it is depicted the battery level of the different nodes using the optimized weights. As it can be observed all nodes deplete their batteries at the same time which is equivalent to maximize the time that the QoS can be satisfied. In Figure 3-b we have an example for a target SNR of $20 \mathrm{~dB}$. We have displayed the achieved SNR as a function of the iteration number for the Consensus Iterative Algorithm 1. It can be appreciated that the algorithm converges to the target SNR of $20 \mathrm{~dB}$. We further illustrate in Figure 3-c and the error between the centralized power allocation and the one achieved using the distributed algorithms. The error term for every node goes to zero as the iteration number increases.

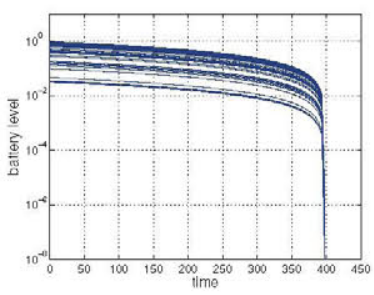

(a) Battery level over time

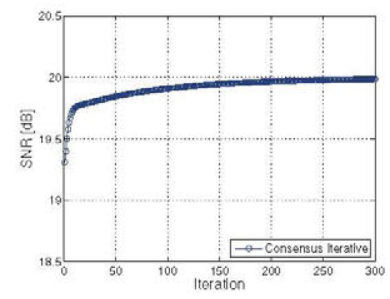

(b) Convergence to the target SNR

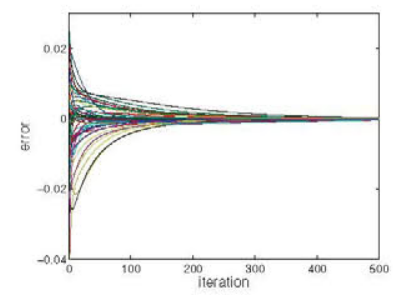

(c) Difference to centralized solution

Fig. 3: Performance of the algorithm

\section{CONCLUSIONS}

We have presented a distributed approach to energy-efficient beamforming in sensor networks. The proposed strategy takes into account the remaining battery level at each node in order to optimize for the network lifetime while guaranteeing a specified QoS requirement. We have validated by means of simulations that the proposed scheme outperforms collaborative beamforming strategy. We have also provided a consensus-based distributed algorithm for the computation of the optimal beamformer.

\section{REFERENCES}

[1] H. Ochiai, P. Mitran, H. V. Poor, and V. Tarokh, "Collaborative beamforming for distributed wireless ad hoc sensor networks," IEEE Transactions on Signal Processing, vol. 53, pp. 4110 4124, 2005.

[2] M. F. A. Ahmed and S. A. Vorobyov, "Performance characteristics of collaborative beamforming for wireless sensor networks with gaussian distributed sensor nodes," ICASSP 2008, pp. 3249-3252.

[3] _ _ "Collaborative beamforming for wireless sensor networks with gaussian distributed sensor nodes," IEEE Trans. Wireless Commun., vol. 8, no. 2, pp. 638-643, 2009.

[4] K. Zarifi, S. Affes, and A. Ghrayeb, "Distributed beamforming for wireless sensor networks with random node location," ICASSP 2009, pp. 2261-2264.

[5] D. P. Palomar and Y. C. Eldar, Convex Optimization in Signal Processing and Communications. Cambridge University Press, 2010.

[6] Z. Han and H. V. Poor, "Lifetime improvement in wireless sensor networks via collaborative beamforming and cooperative transmission," IET Microwaves, Antennas \& Propagation, vol. 1, no. 6, pp. 11031110, 2007.

[7] J. Feng, C.-W. Chang, S. Sayilir, Y.-H. Lu, B. Jung, D. Peroulis, and Y. C. Hu, "Energy-efficient transmission for beamforming in wireless sensor networks," SECON 2010, pp. 1-9.

[8] R. Olfati-Saber and R. Murray, "Consensus Problems in Networks of Agents with Switching Topology and Time-Delays," IEEE Trans. Autom. Control, vol. 49, no. 9, pp. 1520-1533, September 2004.

[9] I. Dietrich and F. Dressler, "On the lifetime of wireless sensor networks," ACM Trans. Sen. Netw., vol. 5, pp. 5:1-5:39, February 2009. [Online]. Available: http://doi.acm.org/10.1145/1464420.1464425 